\title{
28 Research Square \\ Efpeglenatide potentially offers weight-loss treatment option for adults without diabetes
}

\author{
Richard E. Pratley \\ Jahoon Kang \\ Michael E. Trautmann \\ Marcus Hompesch \\ OakPil Han \\ John Stewart \\ Christopher H. Sorli \\ Stephen Jacob \\ Kun-Ho Yoon
}

\section{Video Abstract}

Keywords: obesity, overweight, efpeglenatide, GLP-1 RA, glycemic control, placebo, diabetes, weight loss, drug treatment, dosing, dyslipidemia, hypertension, glucose intolerance, sleep apnea, BMI, FDA, blood lipids, LDL, triglycerides, at-risk patients

Posted Date: October 24th, 2019

DOI: https://doi.org/10.21203/rs.2.16600/v1

License: (c) (i) This work is licensed under a Creative Commons Attribution 4.0 International License. Read Full License 


\section{Abstract}

Losing weight through lifestyle interventions alone can be challenging. Unfortunately, the options for effective drug treatment are limited. And those that are available require frequent, daily dosing. Now, a new treatment option tested among patients with diabetes could be a solution for those with obesity but not diabetes. Shown to significantly reduce body weight vs. placebo, efpeglenatide is one of the latest of an entire class of diabetes-targeted drugs observed to have beneficial effects beyond glycemic control. Efpeglenatide is a long-lasting glucagon-like peptide-1 receptor agonist, or GLP-1 RA. The drug is currently being developed to improve glycemic control in patients with type 2 diabetes as a once-weekly subcutaneous administration. But like other GLP-1 RAs, efpeglenatide also helps patients lose weight. To assess the efficacy, safety, and tolerability of efpeglenatide among adults with obesity or overweight but not diabetes, researchers tested the drug at 4 different doses or frequencies of administration over the course of 20 weeks: once weekly at 4 or $6 \mathrm{mg}$ and once every two weeks at 6 or $8 \mathrm{mg}$. Patients had fasting plasma glucose levels below $7 \mathrm{mmol} / \mathrm{L}$ and BMls of at least $30 \mathrm{~kg} / \mathrm{m} 2$. Patients with lower BMls -at least $27 \mathrm{~kg} / \mathrm{m} 2$-were also included if they showed a comorbidity such as dyslipidemia, hypertension, glucose intolerance, or sleep apnea. Efpeglenatide administered once weekly or once every two weeks led to significant reductions in body weight vs. placebo over the entire treatment period. After 20 weeks, nearly $50 \%$ of all efpeglenatide-treated patients lost at least $5 \%$ of their baseline body weight-with about $20 \%$ losing at least $10 \%$ of their starting body weight. Although a much longer study period of at least one year is needed for confirmation, these initial results suggest that efpeglenatide could meet the FDA's regulatory requirements for effective weight-loss products. Treatment with efpeglenatide also led to significant improvements in other metabolic parameters, including glucose metabolism and lipid profiles. All doses of efpeglenatide significantly reduced fasting glucose and $\mathrm{HbA1c}$ levels compared with placebo. And for most doses of efpeglenatide, significant reductions in blood lipids were observed, including total, LDL, and triglycerides. Most treatment emergent adverse events were of mild or moderate intensity and gastrointestinal in nature, consistent with the effects commonly observed among GLP-1 RAs. More investigations with larger samples and a longer treatment period are needed to confirm the benefits of efpeglenatide. But these preliminary findings suggest that efpeglenatide could be a powerful weight-loss option for at-risk patients with obesity or overweight for whom lifestyle interventions are simply not enough. 